\title{
Erratum to: Preparing Students for Middle School Through After-School STEM Activities
}

\author{
Nancy P. Moreno ${ }^{1} \cdot$ Barbara Z. Tharp ${ }^{1} \cdot{\text { Gregory } \operatorname{Vogt}^{1}}^{1}$ Alana D. Newell ${ }^{1}$. \\ Christopher A. Burnett ${ }^{1}$
}

Published online: 7 November 2016

(C) Springer Science+Business Media New York 2016

Erratum to: J Sci Educ Technol

DOI: $10.1007 / \mathbf{s} 10956-016-9643-3$

The original version of this article unfortunately contained a mistake.

Acknowledgments should read:

The authors express their gratitude to two anonymous reviewers, guest editor Parker and Mr. James Denk for providing careful and thoughtful guidance. Work reprinted here was supported partially by grant number DRL1028771 from the National Science Foundation. Any opinions, findings, and conclusions or recommendations expressed in this material are those of the author(s) and do not necessarily reflect the views of the National Science Foundation. This work was conducted under Human Subjects Research Protocol 1427402, approved by the Institutional Review Board, Baylor College of Medicine.

The online version of the original article can be found at http://dx.doi. org/10.1007/s10956-016-9643-3

Alana D. Newell

adnewell@bcm.edu

1 Baylor College of Medicine, Houston, TX, USA 